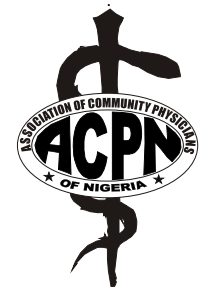

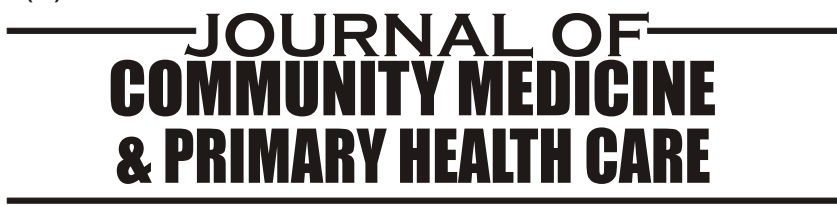

\title{
Compliance to medication among hypertensive patients in Murtala Mohammed Specialist Hospital, Kano, Nigeria
}

\author{
M. Kabir ${ }^{a}$, Z. Iliyasu ${ }^{\text {a }}$, I. S. Abubakar ${ }^{a}$ and M. Jibril' \\ Department of Community Medicine, Aminu Kano Teaching Hospital, Kano, Nigeria and \\ ${ }^{b}$ Department of Community and Primary Care, Bayero University, Kano
}

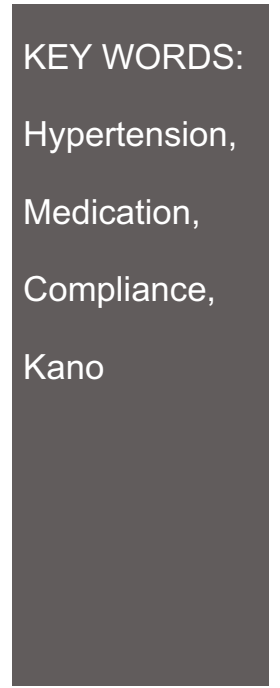

\begin{abstract}
Background: Non-compliance to blood pressure-lowering medication is a major reason for poor control of hypertension worldwide. We assessed the level of compliance to anti-hypertensive therapy and identified factors contributing to poor compliance among hypertensives in Kano.

Methodology: Three hundred and sixty outpatients were interviewed using a pre-tested, structured, mostly closed ended questionnaire in Murtala Mohammed Specialist Hospital in Kano, Nigeria.
\end{abstract}

Results: Good compliance with drug treatment was observed in $54.2 \%$ of the respondents and poor compliance among the remainder. Poor compliance was found to be mainly due to ignorance on need for regular treatment $(32.7 \%)$, lack of funds to purchase drugs $(32.7 \%)$ and side effects of drugs $(12.1 \%)$. Patients with formal education, and higher monthly income were more compliant to treatment. In addition, those on single drugs were more compliant compared to those on two or more drugs. Poor compliance was found to be mainly due to ignorance and lack of funds to purchase drugs.

Conclusion: Based on the findings of this study, there is a need for launching a comprehensive approach involving health care providers, patients and the general public to educating patients on the need to take their drugs regularly and in the manner prescribed. Doctors should consider the financial status of their patients in prescribing antihypertensive drugs to enable affordability. Prices of anti-hypertensive drugs should be subsidized where possible. Prescribing an effective, inexpensive, single dose daily medication with minimal side effects will improve patient compliance considerably.

\section{Introduction}

Developing countries undergoing epidemiological transition face the double burden of communicable and non-communicable diseases. Of the latter, hypertension is one of the most important treatable causes of mortality and morbidity. Although safe and effective drugs are available, the management of hypertension is still far from optimal especially in the developing countries ${ }^{1}$. A major factor accounting for inadequate treatment of hypertension is poor compliance. ${ }^{1,2}$ Compliance can be defined as the degree to which the patient conforms to medical advice about lifestyle and dietary changes as well as to keeping appointments for follow up and taking treatment as prescribed. This can be expressed quantitatively as the percentage of prescribed doses that have been taken. Compliance can be evaluated in several ways ${ }^{4}$. These include pharmacological measures (determination of serum and urinary concentrations of drugs or using biological markers integrated into the tablets); clinical measures (clinical judgement of the doctor, evaluation of promptness for appointments and the use of questionnaires); and

Correspondence to: Dr. Mohammed Kabir

Dept. of Community Medicine

Aminu Kano Teaching Hospital, Kano, Nigeria

E-mail:mdkabir@ecnx.net physical measures (verifying prescription renewals, counting the remaining pills or pill counting systems). Although, there is no gold standard allowing precise measurement of compliance ${ }^{5}$, the electronic pill counter or MEMS (Medication Event Monitoring System) may be considered as the best existing system for measurement of compliance ${ }^{6}$. Pharmacological methods also have a higher sensitivity and specificity ${ }^{7}$ but remain difficult to use in standard practice.

Poor compliance is especially common when a patient has poor knowledge, understanding, and perception of hypertension or when a complex anti-hypertensive drug regime is prescribed ${ }^{8}$. It is usual to consider patients to be sufficiently compliant with their treatment when they take at least $80 \%$ of their prescribed anti-hypertensive drugs $3,9,10$. In outpatients, compliance to anti-hypertensive medication ranges from $20 \%$ to $80 \%$. ${ }^{11-13}$

Nigeria has a high prevalence of hypertension in Nigeria with Kano having the highest prevalence rates. The observed high incidence of complications even among those on treatment due to poor compliance, explain the need for more awareness and 
immediate adjustment in the management pattern with emphasis on compliance ${ }^{2}$.

The aim of this study was to assess the level of compliance to anti-hypertensive therapy and identify factors, which contribute to poor treatment compliance among hypertensives in Kano.

\section{Methodology}

\section{Study setting}

Murtala Mohammed Specialist Hospital, Kano, owned by Kano State is the largest state governmentowned hospital in northern Nigeria and has the highest patients' attendance in the region. Medical, surgical, obstetric, laboratory, radiology and physiotherapy services are offered in the different departments of the hospital. The hypertension clinic is run once in a week for male patients and once in a week for female patients by medical doctors in the department of Medicine. The hospital serves mainly residents in Kano, especially those from the metropolis. Patients from neighbouring states and countries such as Katsina state, Jigawa state, Zamfara state, Niger republic and Mali also attend the hospital.

\begin{abstract}
Methods
A cross-sectional study design was used to study a population of adult patients attending the hypertension clinic of Murtala Specialist Hospital, Kano from $3^{\text {rd }}$ May to $28^{\text {th }}$ June, 2004. A sample size of 360 was obtained using an appropriate statistical formula for estimating minimum sample size in health studies $^{14}$ and the prevalence of poor compliance as found in a study on awareness of hypertension in a Nigerian tertiary health institution ${ }^{15}$. The sample selection was done over a period of six weeks with 30 patients selected by systematic sampling from a listing on each of the clinic days for male and female patients until 180 male and the same number of female patients were selected.
\end{abstract}

The selected patients were interviewed using a pretested, structured, mostly closed- ended questionnaire after obtaining informed consent from the participants. Information obtained included sociodemographic characteristics of respondents, regularity of patient's attendance at the hypertension clinic; patient's reported compliance with prescribed drugs and reasons for non compliance. Patients' compliance were classified using the following criteria $^{4}$ : good compliance included regular attendance at follow-up clinics and obtaining and taking all drugs prescribed, taking the drugs in a day as prescribed, taking drugs for at least 6 to 7 days in a week. Poor compliance referred to irregular attendance at follow-up clinics, inability to obtain drugs regularly, not taking drugs in a day as prescribed, taking drugs for less than 6 days in a week. The fourth section asked about factors militating against good compliance.

Data was analysed using Epi-Info version 6.0 statistical software (CDC Atlanta, Georgia, U.S.A). Absolute numbers and simple percentages were used to describe categorical variables. Similarly, quantitative variables were described using measures of central tendency (mean, median) and measures of dispersion (range, standard deviation) as appropriate. The Chi-square test was used in assessing the significance of associations between categorical groups. A p-value of 0.05 or less was considered statistically significant.

\section{Results}

The ages of respondents ranged between 20 to 94 years with a mean age (SD) of 533.6 years. The majority of respondents (49.4\%) were between $50-64$ years of age.

There were equal number of men and women in the study. Majority of respondents (88.6\%) lived within Kano metropolis while the rest lived in nearby rural areas or came from neighbouring states. Only $27 \%$ of respondents had formal education (primary, secondary and tertiary education) and majority of them earned less than $\mathrm{N} 10,000$ (about twice the national minimum wage) per month. The Hausa ethnic group constituted the majority $(82.5 \%)$ of the respondents, followed by Fulani $(9.2 \%)$, Kanuri $(4.7 \%)$, Yoruba $(1.7 \%)$ and lgbo $(0.5 \%)$. The remaining $(1.9 \%)$ were from other minority Nigerian tribes including Tiv, Egbira and Babur. Most of the respondents (73.6\%) were married while the rest were widowed, divorced or separated. Only $0.8 \%$ was single. Majority $(97.5 \%)$ of the patients were Muslims while the remaining $2.5 \%$ were Christians.

Table I shows that $54.2 \%$ of respondents had good compliance with treatment whereas the remaining $45.8 \%$ had poor compliance. Majority of the patients were regular clinic attendees while a quarter were irregular as shown in Table 2.

Poor compliance was found to be mainly due to ignorance on need for regular treatment $(32.7 \%)$ and lack of funds to purchase drugs $(32.7 \%)$. Other factors included side effects of drugs (12.1\%), non-availability of drugs in patients' place of domicile $(8.0 \%)$, exhaustion of prescribed drugs and non-attendance at scheduled clinic day (4.8\%), normal blood pressure during the previous clinic visit $(3.6 \%)$, forgetfulness (3.0\%), and busy schedule $(1.8 \%)$.

Seventy two $(74.2 \%)$ of the 97 respondents who had formal education were compliant with their treatment compared to only 123 of the $263(47.0 \%)$ respondents without formal education. This difference was statistically significant $\left(\mathrm{X}^{2}=21.5 \mathrm{df}=1 \quad \mathrm{P}<0.01\right)$. Similarly, a higher proportion $113(89.7 \%)$ of those with monthly income of above 10, 000 Naira were compliant with their treatment compared to $82(35.0 \%)$ of those whose monthly income was less than 10,000 
whose monthly income was less than 10,000 Naira $\left(X^{2}=98.5 \mathrm{df}=1 \mathrm{P}<0.01\right)$. Similarly, a significantly higher proportion 97 (84.3\%) of patients who were on one or two types of drugs showed better compliance compared to $98(40.0 \%)$ of those being treated with more than two types of drugs $\left(X^{2}=61.9\right.$, df $=1 P$ $<0.01)$. drugs. Once daily regimens was found to have better compliance than three times daily ones. Furthermore, medication compliance was greatest for morning dosing, second greatest for evening doses and least for noon. This could be explained by the fact that patients are usually at home and less busy during the peak compliance periods as opposed to midday. In Bangladesh and India on the other hand a WHO

\begin{tabular}{lll} 
Table I: & \multicolumn{3}{l}{ Level of compliance with drugs among hypertensive patients } \\
\cline { 2 - 3 } Compliance level & Frequency & Percentage (\%) \\
\hline Poor & 165 & 45.8 \\
Good & 195 & 54.2 \\
\hline Total & 360 & 100 \\
\hline
\end{tabular}

\section{Discussion}

This study revealed that almost half of the hypertensive patients in Murtala Mohammed specialist hospital had poor compliance with respect to drug treatment. Isezuo and Opara reported similar findings in a study on awareness of hypertension in Usman Danfodio teaching hospital Sokoto in northern Nigeria ${ }^{15}$. Furthermore, Olubodun and colleagues $^{19}$ reported low compliance among all socio-economic groups in Ibadan. They found that $59.5 \%$ of the patients were not informed by their physicians of the need for continued and regular treatment and follow-up. In another study in Seychelles to monitor one-year compliance to anti hypertension medication in a general hospital setting study found satisfactory compliance in only $25 \%$ of patients ${ }^{18}$. These findings seem to suggest that compliance is not unrelated to awareness and standard of living of people. Our finding of better compliance among the educated and those with higher incomes tend to support this point.

Majority of the patients in this study attend hypertension clinic regularly. The observed good compliance among regular clinic attendees is not surprising since they are also more likely to follow other instructions.

The observed association between poor compliance, ignorance and lack of funds for the purchase drugs were also reported by Isezuo and Opara as being

Table 2: Regularity of clinic attendance among hypertensive patients

\begin{tabular}{lcl}
\hline Regularity & Frequency & Percentage (\%) \\
\hline Regular & 267 & 74.2 \\
Not regular & 93 & 25.8 \\
\hline Total & 360 & 100.0 \\
\hline
\end{tabular}

the prevalence of poor compliance was found to be $46 \%{ }^{17}$ But in a study carried out in a university hospital in Switzerland, poor compliance was observed in only $20.2 \%$ of patients ${ }^{16}$. Similarly, a Japanese study ${ }^{15}$ found that $63.8 \%$ of their patients reported taking $95-100 \%$ of prescribed drugs. Medication compliance was related to the frequency and timing of dosing rather than to the number of responsible for poor compliance in a study among hypertensive patients in Usman Danfodio teaching hospital, Sokoto. ${ }^{15}$ Other factors not as important as these first two include side effects of drugs, nonavailability of drugs in patients' place of domicile, exhaustion of prescribed drugs before the next appointment, normal blood pressure during the previous clinic visit, forgetfulness, and busy 
Table 3: Factors responsible for poor drug compliance among patients $\mathrm{n}=165$

\begin{tabular}{lcc}
\hline Factors responsible for & Frequency & Percentage (\%) \\
Poor compliance & 56 & 34.0 \\
\hline & 54 & 32.7 \\
Absence of symptoms & 20 & 12.1 \\
Lack of funds to purchase drugs & 13 & 8.0 \\
Side effect of drugs & 8 & 4.8 \\
Non-availability of drugs & 6 & 3.6 \\
Exhaustion of prescribed drugs & 5 & 3.0 \\
Normal blood pressure during & 3 & 1.8 \\
$\quad$ Previous clinic visit & & 100 \\
Borgetfulness & 165 & \\
\hline Tusy schedule & & \\
\hline
\end{tabular}

responsible for poor compliance in a study among hypertensive patients in Usman Danfodio teaching hospital, Sokoto. ${ }^{15}$ Other factors not as important as these first two include side effects of drugs, nonavailability of drugs in patients' place of domicile, exhaustion of prescribed drugs before the next appointment, normal blood pressure during the previous clinic visit, forgetfulness, and busy schedule. The lower compliance among those on more than two medications compared to those on fewer medications has also been reported by other workers $^{16}$. The report from Ibadan ${ }^{19}$ also identified economic constraints, use of alternative treatment, frustrations with daily drug ingestion and feeling of well being as impediment to compliance. Similar findings were reported from studies of compliance among hypertensives in Switzerland ${ }^{16}$ and Seychelles $^{17}$ respectively. Schroeder and colleagues $^{21}$ found during a systematic review of effective interventions for improved compliance that simplifying dosage regimens, improved doctorpatient communication and motivational strategies in combination was more effective than patient education alone. Similarly, Moore ${ }^{22}$ reported that optimal control of hypertension was impeded by poor patient-physician communication, excessive dietary sodium, and cost of anti-hypertensive medication and laboratory studies.

Based on the findings of this study there is a need for launching a comprehensive approach involving health care providers, patients and the general public especially with the aim of educating patients on the need to take their drugs regularly and in the manner prescribed. Doctors should consider the financial status of their patients in prescribing antihypertensive drugs to enable affordability. Prizes of anti-hypertensive drugs should be subsidized where possible. Prescribing an effective, inexpensive, one daily medication with minimal side effects will improve patient compliance considerably.

\section{References}

1. Luscher T.F., Vetter H., Siegenthaler W and Vetter W., Compliance in hypertension: facts and concepts. $J$ Hypertens Suppl. 1985 Apr; 3(1): 3-9.

2. Federal Ministry of Health (Nigeria). Noncommunicable disease in Nigeria: Final report of a national survey by the National Expert Committee on Non-communicable diseases 1997:14-41

3. Guerrero D, Rudd P, Bryant-Kosling C, Middleton BF. Antihypertensive

medication-taking: investigation of a simple regimen. Am J Hypertens 1993;

6:586-92.

4. Garfield FN, Caro JJ. Cmpliaance and hypertension. Current Hypertension

Reports 1999; 1: 502-6.

5. Mallion J.M., Schmitt D. Patient compliance in the treatment of arterial hypertension. European Society of Hypertension Scientific Newsletter. 2001; 2:7

6 . Eisen SA, Woodward RS, Miller D, Spitznagel E, Windham CA. The effect of

medication compliance on the control of hypertension. J Gen Intern Med 1987; 2(5): 298-305.

7. Bittar N. Maintaining long-term control of blood pressure: the role of

improved compliance. Clin Cardiol 1995; 18: III12III16.

8. Martin $U$ and Nwankwo L. Blood pressure in Nigerian College males. Journal of Human hypertension 1990; 4: 72-3

9. Krall R. Interactions of compliance and patient safety. In: Patient compliance in medical practice and clinical trials. New York: Raven press. 1991:19-25.

10. Sackett D., Haynes R., Gibson E., Taylor D., Roberts $\mathrm{R}$ and Johnson A. Patient compliance with antihypertensive regimens. Patient Couns Health Educ 1978; 1:18-21

11. Sixth report of the Joint National Committee on Prevention, Detection, Evaluation and treatment of high blood pressure. Archives of Internal Medicine 1997; 157:2413-46 
12. Cramer J.A. Consequences of Intermittent treatment for hypertension: the case for medication compliance and persistence. American Journal of Managed Care 1998; 4: 1563-8

13. Waeber B., Vetter W., Darioli R., Keller U., Brunner H.R. Improved blood pressure control by monitoring compliance to antihypertensive therapy. International Journal of Clinical Practice 1999; 53:37-8

14. Lwanga S., Lemeshow S., Sample size determination in health studies: a practical manual. Geneva, World health Organization. 1991:24-5

15. Isezuo A.S., Opera T.C. Hypertension awareness among Nigerians in a Nigerian tertiary health institution. Sahel Medical Journal 2000; 3(2): 93-96 16. Muesch R., Schroeder K., Dieterle T., Martina B and Battegay E., Relationship between insufficient response to antihypertensive treatment and poor compliance with treatment; a prospective case control study. BMJ 2001; (32): 143-5

17. Bovet P., Burnier M., Mandeleine G., Waeber B., and Paccaud F., Monitoring one year compliance to antihypertensive medication in the Seychelles. Bulletin of the World Health Organization 2002; 80(1): 33-8
18. Thankappan K.R and Hypertension study group. Hypertension among the elderly in Bangladesh and India: a Multicentre study. Bulletin of the World health Organization 2001; 79(60): 490-9

19. Olubodun J.O., Falase A.O and Cole T.O. Drug compliance in hypertensive Nigerians with and without heart failure. Int J Cardiol. 1990 May; 27(2): 229-34

20. Fujii J., Seki A. Compliance and complianceimproving strategies in hypertension: the Japanese experience. J Hypertens Suppl. 1985 Apr; 3(1): S1922

21. Schroeder K., Fahey T and Ebrahim S. How can we improve adherence to blood pressure-lowering medication in ambulatory care? Systematic review of randomized controlled trials. Arch Intern Med. 2004 Apr 12; 164 (7): 722-32

22. Moore M.A. Improving compliance with antihypertensive therapy. Am Fam Physician. 1988 Jan; 37 (1): 142-8 